\title{
PHÂN TÍCH KÍCH THƯớC KHUÔN MẶT THEO HÌNH DẠNG KHUÔN MẶT NGƯờI VIÊTT TỪ 18 ĐẾN 25 TÛ̂̉I TẠI TỈNH BÌNH DƯƠNG
}

\author{
Võ Trương Như Ngọc**, Nguyễn Hữu Huynh*
}

\section{TÓM TẮT}

Nghiên cứu được thực hiện trên 2462 người Việt dân tộc Kinh tuổi từ 18 đến 25 (1328 nữ, 1134 nam) nhằm mục tiêu so sánh một số kích thước khuôn măt theo các kiểu hình dang khuôn măt trên ảnh chuẩn hóa củangười Việt tuổi từ 18 đến 25 tại tỉnh Bình Dương. Đối tượng và phương pháp nghiên cứu: nghiên cứu được thực hiện theo phương pháp mô tả cắt ngang trên. Kết quả: khuôn mă̆t hình oval chiếm tỉ lệ cao nhất $(66,3 \%)$, khuôn mặt hình vuông $(26,6 \%)$, thấp nhất là khuôn mặt hình tam giác $(7,1 \%)$; kích thước chiều rộng thái dương (Ft-Ft), chiều rộng mặt $(Z y-Z y)$, chiêu rộng hàm dưới (Go-Go) ở ba dạng mặt có sự khác biệt có ý nghĩa thống kê, sáu kích thước ngang còn lại khác biệt không có ý nghĩa thống kê. Kết luận: khuôn mặt hình oval chiếm tỷ lệ cao nhất, ít hơn là mặt hình vuông và ít nhất là mặt hình tam giác; ít có sự khác biệt giữa hầu hết các kích thước ngang giữa ba dạng khuồn mătt.

Từ khóa: nhân trắc học, ảnh chuẩn hóa, hình dạng khuôn mặt, kích thước ngang

\section{SUMMARY}

\section{ANALYSE SOMES DIMENSIONS FOLLOWINGVIETNAM FACE SHAPE FROM 18 TO 25 YEARS OLD IN BINH DUONG PROVINCE}

Purpose:This research have carried out to analyse somes dimensions following the face shapes on standardized images of Vietnamese aged 18 to 25 in Binh Duong province. Participants and methods: Cross-sectional studies on 2462 Kinh Vietnamese people aged 18 to 25 (1328 female, 1134 male), using standardized image of straight posture to determine the facial horizontal dimensions. Results: oval face accounted for the highest proportion $(66.3 \%)$, square face $(26.6 \%)$, the lowest was triangular face $(7.1 \%)$; the bifrontotemporal width (Ft-Ft), bizygomatic breadth (Zy-Zy), and bigonial breadth (Go-Go) in three faces have statistically significant differences, the remaining six horizontal dimensions that were not statistically significant differences. Conclusions: oval face has the highest proportion, following by square face and least triangular face; there is little difference between most horizontal dimensions between the three face types.

Keywords: Anthropometric, photogrammetry, face shape,

\footnotetext{
*Viện đào tạo Răng Hàm Mặt- Trường Đại học Y Hà Nội Chịu trách nhiệm chính: Võ Trương Như Ngọc Email: votruongnhungoc@gmail.com Ngày nhận bài: 5.3.2021

Ngày phản biên khoa học: 22.4.2021

Ngày duyệt bài: 28.4.2021
}

\section{I. ĐẠT VẤN ĐỀ}

Nhân trắc học là khoa học đóng góp phần quyết định trong việc nghiên cứu sư phát triển của cơ thể con người theo từng thời kỳ. Bằng phương pháp nhân trắc học, người ta có thể so sánh, đánh giá về hình thái và sư tăng trưởng bình thường hay bất thường mỗi con người. Những hằng số nhân trắc cho ta những chỉ số mang tính chất tham khảo, ứng dụng nhiều trong y học. Để phân tích sự khác nhau về hình thái khuôn mặt thì phương pháp phân tích gián tiếp qua ảnh có wuu điểm là nhanh gọn, thu thập được số lượng mẫu lớn với thời gian ngắn, chi phí thấp...được sử dụng như một công cụ̣ thiết yếu trong chỉnh hình răng và phẫu thuật tạo hình hàm mặt hay phẫu thuật thẩm mỹ ${ }^{1,2}$.X̃̃ hội đang ngày càng phát triển thì nhu cầu thẩm mỹ nói chung và thẩm mỹ khuôn mặt nói riêng ngày càng cao. Do vâyy, thẩm mỹ về khuôn mặt là một trong những vấn đề nghiên cứu được ưu tiên hàng đâu ${ }^{2}$. Tại Việt Nam các nghiên cứu về đặc điểm nhân trắc được tiến hành trên đối tượng hạn chế và chưa được hệ thống hóa để xác định tiêu chuẩn đánh giá khuôn mặt hài hòa.Cho đến nay chưa có một hệ thống chỉ số, hằng số trung bình dành cho người Việt được nghiên cứu trên cộng đồng với số lượng đủ. Vì vậy, chúng tôi tiến hành đề tài này với mục tiêu mô tả tương quan kích thước với các kiểu hình dạng khuôn mặt trên ảnh chuẩn hóa của người Việt tuổi từ 18 đến 25 tại tỉnh Bình Dương.

\section{II. ĐỐI TƯỢNG VÀ PHƯƠNG PHÁP NGHIÊN CỨU}

2.1. Đối tượng nghiên cứu: 2462 người Việt (1328 nữ, 1134 nam) dân tộc Kinh tuổi từ 18 đến 25 đang sống tại tỉnh Bình Dương.

Tiêu chuẩn lựa chọn: là người khỏe mạnh, có bố mẹ, ông bà nội ngoại là người Việt, không có dị dạng hàm mặt, không có tiền sử chấn thương hay phẫu thuật vùng hàm mặt, chưa điều trị nắn chỉnh răng và các phẫu thuật tạo hình khác, không có biến dạng xương hàm, có đủ răng, đồng ý và tự nguyện tham gia nghiên cứu.

Tiêu chuẩn loại trừ: là những người không đạt được tiêu chuẩn lựa chọn trên.

\subsection{Phương pháp nghiên cứu}

Thiết kế nghiên cứu: nghiên cứu mô tả cắt ngang.

Cỡ mẫu: sử dụng công thức tính cõ̃ mẫu ước 
tính một chỉ số trung bình cho nghiên cứu điều tra cắt ngang, tính được cỡ mẫu tối thiểu cho nghiên cứu là 1985 người.

Chọn mẫu: ngẫu nhiên đơn.

Thời gian và địa điểm nghiên cứu: từ tháng 9/2017 đến tháng 09/2018 tại các trường Đại học và Cao đẳng trên địa bàn tỉnh Bình Dương.

Các bước tiến hành:Tất cả các đối tượng nghiên cứu được chụp ảnh chuẩn hóa theo tư thễ thẳng. Phương tiện nghiên cứu: máy ảnh Nikon D700, ống kính tiêu cự cố định $105 \mathrm{~mm}$, khoảng cách từ đối tượng đến cảm biến máy ảnh $1,5 \mathrm{~m}$. Tư thế chụp: ngồi thẳng, đầu ở tư thế tự nhiên, mắt nhìn thẳng. Ảnh được số hóa và mã hóa theo số thứ tự. Ảnh được chuẩn hóa kích thước và được đánh dấu các điểm mốc bằng phần mềm đo đạc ảnh VNCEPH (giấy phép chứng nhận số 5138/2017/QTG).

Một sổ điểm mốc được đánh dấu trên ảnh thẳng chuẩn hóa: điểm khóe mắt trong (En), điểm khóe mắt ngoài $(E x)$, điểm cánh mũi $(\mathrm{Al})$, điểm gò má $(Z y)$, điểm khóe miệng $(\mathrm{Ch})$, điểm sau nhất và dưới nhất của góc hàm dưới (Go), điểm chính giữa đồng tử (Pp), điểm thái dương $(\mathrm{Ft})$.

Các kích thước ngang của khuôn mặt: kích thước chiều rộng thái dương (Ft-Ft), chiều rộng mặt (Zy-Zy), chiêuu rộng hàm dưới (Go-Go), kích thước chiêu rộng mũi $(\mathrm{Al}-\mathrm{Al})$, chiều rộng mắt (En-Ex), chiều rộng miệng (Ch-Ch) khoảng cách giữa hai góc mắt trong (En-En), khoảng cách từ góc miệng đến đồng tử cùng bên (Ch-Pp), khoảng cách từ mũi đến khóe miệng ( $\mathrm{Al}-\mathrm{Ch}$ ).

Phân loại hình dáng khuôn mặt theo Celébie và Jerolimov dựa vào khoảng cách $\mathrm{Ft}-\mathrm{Ft}, \mathrm{Zy}-\mathrm{Zy}$ và Go-Go: mặt hình vuông nếu Go-Go $=Z y-Z y=$ Ft-Ft hoặc Ft-Ft = Zy-Zy hoặc Zy-Zy = Go-Go, mặt hình oval nếu Zy-Zy > Ft-Ft và Zy-Zy >GoGo, mặt hình tam giác nếu Ft-Ft $>Z y-Z y>$ GoGo hoặc Ft-Ft < Zy-Zy < Go-Go (nếu 2 kích thước chênh nhau khoảng $2 \mathrm{~mm}$ thì coi như là bằng nhau).

Xử lý số liệu: Số liệu kích thước ngang khuôn măt sau khi xuất ra từ phần mềm VNCEPH sẽ được xử lý, phân tích kết quả bằng phần mềm SPSS 16.0 sử dụng kiểm định phi tham số Kruskal-Wallis test để kiểm định so sánh, chọn có ý nghĩa thống kê với $p<0.05$.

2.3. Đaao đức trong nghiên cứu: nghiên cứu này tuân thủ đầy đủ các nguyên tắc đạo đức của đề tài cấp Nhà nước: "Nghiên cứu đặc điểm nhân trắc đầu mặt ở người Việt Nam để ứng dung trong y học" đã được chấp thuận bởi Hội đồng Đạo đức trong nghiên cứu $Y$ sinh học Trường Đại học $Y$ Hà Nội theo quyết định số 202/HĐĐĐĐHYHN ngày 28/10/2016.

\section{KẾT QUẢ NGHIÊN CứU}

Nghiên cứu được thực hiện trên 2462 người Việt dân tộc Kinh tuổi từ 18 đến 25 (1328 nữ chiếm tỷ lệ $53,9 \%, 1134$ nam chiếm tỷ lệ $46,1 \%$ )

Phân loại hình thái mặt theo Celébie và Jerolimov trên ảnh chuẩn hóa

Tỷ lệ hình dạng khuôn mặt

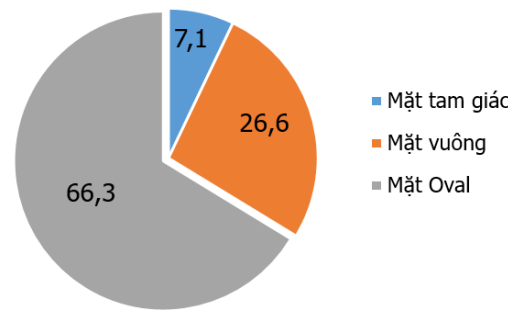

\section{Biểu đồ 3.2. Phân bố đôî tượng theo phân loại hinh thái khuôn mặt của Celébie và Jerolimov}

Nhận xét: Tỉ lệ đối tượng có khuôn mặt hình Oval chiếm tỉ lệ cao nhất $(66,3 \%)$, thấp nhất là khuôn mặt hình tam giác $(7,1 \%)$ và còn lại là hình Vuông (26,6\%).

So sánh tương quan giữa các kích thước theo hình dạng mặt

Bảng 3.1. So sánh các kích thước theo hình dang mắt

\begin{tabular}{|c|c|c|c|c|c|}
\hline \multirow{2}{*}{ Phép đo } & & \multicolumn{3}{|c|}{ Hình dạng mặt } & \multirow{2}{*}{$\mathbf{P}$} \\
\hline & & Mặt tam giác & Mặt vuông & Mặt Oval & \\
\hline \multirow{2}{*}{ En-En } & $\mathrm{X} \pm \mathrm{SD}$ & $38,59 \pm 3,45$ & $38,59 \pm 3,71$ & $38,7 \pm 3,64$ & \multirow[b]{2}{*}{$>0,05$} \\
\hline & $-N$ & 175 & 654 & 1633 & \\
\hline \multirow{2}{*}{ Al-Al } & $\overline{\mathrm{X}} \pm \mathrm{SD}$ & $41,34 \pm 3,87$ & $41,97 \pm 3,9$ & $41,89 \pm 3,85$ & \multirow{2}{*}{$>0,05$} \\
\hline & $\mathrm{N}$ & 175 & 654 & 1633 & \\
\hline \multirow[b]{2}{*}{ Ex-En } & $X \pm S D$ & $29,51 \pm 2,92$ & $29,6 \pm 3,31$ & $29,68 \pm 3,13$ & $>0,05$ \\
\hline & $\mathrm{N}$ & 175 & 654 & 1633 & \multirow{3}{*}{$>0,05$} \\
\hline \multirow{2}{*}{ Ch-Ch } & $\bar{X} \pm \mathrm{SD}$ & $48,87 \pm 4,93$ & $49,13 \pm 4,91$ & $49,11 \pm 4,83$ & \\
\hline & $\mathrm{N}$ & 175 & 654 & 1633 & \\
\hline \multirow{2}{*}{$Z y-Z y$} & $\mathrm{X} \pm \mathrm{SD}$ & $145,02 \pm 8,58$ & $145,98 \pm 9,09$ & $146,27 \pm 8,86$ & \multirow{2}{*}{$<0,05$} \\
\hline & $\mathrm{N}$ & 175 & 654 & 1633 & \\
\hline
\end{tabular}


TẠP CHÍ Y HỌC VIỆT NAM TẬP 502 - THÁNG 5 - SỐ 1 - 2021

\begin{tabular}{|c|c|c|c|c|c|}
\hline \multirow{2}{*}{$\mathrm{Al}-\mathrm{Ch}$} & $\overline{\mathrm{X}} \pm \mathrm{SD}$ & $3,97 \pm 1,96$ & $3,85 \pm 1,86$ & $3,91 \pm 1,86$ & \multirow{2}{*}{$>0,05$} \\
\hline & $-\mathrm{N}$ & 175 & 654 & 1633 & \\
\hline \multirow{2}{*}{ Go-Go } & $\bar{X} \pm S D$ & $122,03 \pm 9,83$ & $123,62 \pm 10,93$ & $123,46 \pm 10,18$ & \multirow{2}{*}{$<0,05$} \\
\hline & $-\mathrm{N}$ & 175 & 654 & 1633 & \\
\hline \multirow{2}{*}{ Ch-Pp } & $\mathrm{X} \pm \mathrm{SD}$ & $8,42 \pm 2,31$ & $8,37 \pm 2,37$ & $8,51 \pm 2,78$ & \multirow[b]{2}{*}{$>0,05$} \\
\hline & $\mathrm{N}$ & 175 & 654 & 1633 & \\
\hline \multirow{2}{*}{ Ft-Ft } & $\bar{X} \pm S D$ & $148,79 \pm 8,85$ & $145,51 \pm 9,19$ & $139,16 \pm 10,26$ & \multirow{2}{*}{$<0,001$} \\
\hline & $\mathrm{N}$ & 175 & 654 & 1633 & \\
\hline
\end{tabular}

Nhận xét: Chỉ có kích thước Zy-Zy, Go-Go và Ft-Ft là có sự khác biệt giữa các hình dạng mặt, còn lại 6/9 kích thước không có sự khác biệt giữa các hình dạng mặt.

\section{BÀN LUẬN}

Nghiên cứu của chúng tôi tiến hành trên 2462 đối tượng độ tuổi $18-25$ chủ yếu là sinh viên và học viên của các trường Đại học và Cao đẳng trên địa bàn tỉnh Bình Dương. Trong đó, nữ giới chiếm $53,9 \%$ cao hơn nam giới 46,1\%.Tất cả các đối tượng nghiên cứu đều là người dân tộc Kinh, có độ tuổi phù hợp với yêu cầu nghiên cứu nhân trắc học và có thể đại diện cho người trưởng thành vì phần lớn các nghiên cứu đều cho rằng phức hợp sọ mặt đều trưởng thành trước tuổi 16. Sự khác biệt không có ý nghĩa thống kê giữa hai giới ${ }^{4,8}$.

Kết quả từ biểu đồ 3.1 cho thãy đa số đối tượng nghiên cứu của chúng tôi có khuôn mặt hình oval, chiếm tỷ lệ cao nhất $66,3 \%$, thấp hởn là khuôn mặt hình tam giác (26.6\%) và ít nhất là khuôn mặt hình vuông $(7,1 \%)$. Sự khác biệt này có ý nghĩa thống kê với $p<0,05$. Kết quả này cũng tương đồng với một số tác giả khác cũng nghiên cứu trên nhóm tuổi này. Cụ thể, nghiên cứu của Võ Trương Như Ngọc ${ }^{5}$ năm 2014 trên sinh viên Trường Đại học $Y$ Hà Nội cho thấy nhiều nhất là khuôn mặt hình oval, sau đó là khuôn mặt hình vuông và ít nhất là khuôn mặt hình tam giác;kết quả này tương đồng với nghiên cứu của Trần Tuấn Anh ${ }^{6}$ năm 2013trên sinh viên Trường Cao đẳng $\mathrm{y}$ tế Bình Dương. Như vậy, có thể thấy đa số thanh niên Việt Nam ở độ tuổi trưởng thành (18-25 tuổi) có khuôn mặt hình oval, ít hơn là khuôn mặt hình vuông và ít nhất là khuôn mặt hình tam giác ${ }^{7}$.

Trong mấu nghiên cứu của chúng tôi, theo bảng 3.1hầu hết các kích thước ngang của các dạng mặt khác nhau không có ý nghĩa thống kê.Khoảng cách giữa hai góc mắt trong (En-En), khoảng cách từ góc miệng đến đồng tử cùng bên (Ch-Pp) khác biêt không có ý nghĩa thống kê.Các kích thước chiều rộng thái dương (Ft-Ft), chiều rộng mặt $(Z y-Z y)$, chiều rộng hàm dưới (Go-Go) ở ba dạng mặt có sự khác biệt có ý nghĩa thống kê. Điều này cũng hoàn toàn phù hợp theo phân loại của Celebie và Jerolimov:
Kiểu mặt hình vuông nếu Go-Go $=\mathrm{Zy}-\mathrm{Zy}=\mathrm{Ft}-\mathrm{Ft}$ hoặc Ft-Ft $=Z y-Z y$ hoặc Zy-Zy = Go-Go, mặt hình oval nếu Zy-Zy > Ft-Ft và Zy-Zy >Go-Go, mặt hình tam giác nếu Ft-Ft $>Z y-Z y>$ Go-Go hoặc Ft-Ft < Zy-Zy < Go-Go.

Các kích thước chiều rộng mũi (Al-Al), chiều rộng mắt (En-Ex), chiều rộng miệng (Ch-Ch) của ba kiểu mặt cũng không có sự khác biệt có ý nghĩa thống kê với $p>0,05$.Chứng tỏ các kích thước ngang chưa có sự đặc trưng giữa các kiểu mặt.

\section{KẾT LUÂNN}

Khi phân tích hình dạng khuôn mặt và so sánh các kích thước giữa các khuôn mặt trên đối tượng thanh niên Việt Nam khỏe mạnh tuổi từ 18-25 với cỡ mẫu đủ lớn, chúng tôi nhận thấy đa số có khuôn mă̆t hình oval, ít hơn là khuôn mặt hình vuôn và rất ít khuôn mặt hình tam giác. Bên cạnh đó, cũng ít có sự khác biệt giữa hầu hết các kích thước ngang giữa ba dạng khuôn mặt.

\section{Lờ' CẢM ƠN}

Để hoàn thành bài báo này, chúng tôi xin gửi lời cảm ơn trân trọng nhất đến các đối tượng nghiên cứu, các thầy cô Viện đào tạo Răng Hàm Mặt - Trường Đại học $Y$ Hà Nội, văn phòng quản lý chương trình trọng điểm quốc giá đã tạo điều kiện hết sức thuận lợi cho nhóm nghiên cứu có thể hoàn thành đề tài này.

\section{TÀI LIÊU THAM KHẢO}

1. Hoàng Tử Hùng, Hồ Thị Thùy Trang (1999). Những đặc trưng của khuổn mặt hài hòa qua ảnh chup và phim sọ nghiêng. Hình thái học, thành phổ Hồ Chí Minh, tập 9, 64-74.

2. Võ Công Minh, Trần Thi Bích Liên (2010). So sánh khuôn mặt người Việ̂t Nam trưởng thành và khuôn mă̆t người Châu Á khác. Tạp Chí Y Học Thành Phố Hồ Chí Minh, 14, 256-261.

3. Kolar, J. C. (1993). Methods in anthropometric studies. The Cleft Palate-Craniofacial Journal: Official Publication of the American Cleft PalateCraniofacial Association, 30(4), 429-431.

4. Milosević, S. A., Varga, M. L., Slaj, M. (2008). Analysis of the soft tissue facial profile of Croatians using of linear measurements. The Journal of Craniofacial Surgery, 19(1), 251-258. 
5. Võ Trương Như Ngọc, Trương Mạnh Dũng, Tống Minh Sơn, Trinh Thi Thái Hà (2014). Nhận xét chuẩn tấn cổ điển ở môt nhóm sinh viên 18-25 có khuôn mặt trên ảnh kỹ̃ thuật số chuẩn hóa. Tap Chí Y Hoc Thức Hành, 3, 24-27.

6. Trân Tưuán Anh, Võ Trương Như Ngọc (2013). Đăc điểm hình thái khuôn mặt ở một nhóm ngướ Việt độ tuổi từ 18-25 tại trường Cao Đẳng $Y$ tế Bình Dương. Tap Chí Y Học Viêtt Nam, 2, 66-75.

7. Võ Trương Như Ngọc, Trương Mạnh Dũng,
Tống Minh Sơn, Tringh Thị Thái Hà (2014), Nhân xét chuẩn tân cổ điển ở một nhóm sinh viên 18-25 tuổi có khuôn măt hài hòa trên ảnh kỹ thuật số chuẩn hóa, Tap chí Y Học Thực Hành, số 2 (906), trang 131-136.

8. Võ Trương Như Ngọc, Nguyễn Đức Nghĩa (2014), Mối tương quan giữa các kích thước khuôn măt trên ảnh chuẩn hóa ở môt nhóm hoc sinh PTTH- Trường Chu Văn An Hà Nội năm 2012, Tap chí Y Hoc Thực Hành, số 2 (906), trang 140-144.

\title{
NGHIÊN CỨU BÀO CHẾ VIÊN NÉN ACID ALPHA LIPOIC 300 MG
}

\author{
Trần Văn Vinh ${ }^{1}$, Nguyễn Văn Giang ${ }^{1}$, Phạm Văn Hùng ${ }^{1}$ \\ Đàm Thanh Xuân ${ }^{1}$, Nguyễn Thị Thanh Duyên ${ }^{1}$
}

\section{TÓM TẮT}

Muc tiêu: (i) Bước đầu đánh giá được ảnh hưởng của một số yếu tố thuộc công thức bào chế đến khả năng khắc phục hiện tượng dính chày cối trong quá trình dâp viên và độ hòa tan (ĐHT) của viên nén acid alpha lipoic (ALA) 300 mg; (ii) Bào chế được viên nén ALA 300 mg đạt tiêu chuẩn ĐHT theo Dược điển Mỹ (USP) 41 ở quy mô phòng thí nghiệm. Phương pháp nghiên cứu: Bào chế viên nén ALA bằng phương pháp tạo hạt ướt và đánh giá ảnh hưởng của một số yếu tố bằng thực nghiêm. Kết quả: Công thức viên nén ALA $300 \mathrm{mg}$ tìm được gồm: ALA: $300 \mathrm{mg}$, Avicel $\mathrm{PH}-102: 120 \mathrm{mg}$, natri stearyl fumarat (NSF): 19,1 mg, Aerosil 200: 19,1 mg, talc: 9,6 mg, dinatri edetat (EDTA): 1mg, hydroxypropyl cellulose (HPC): 12,2 mg, natri croscarmellose (NCC): 25 mg, menthol: 12 mg, poloxamer: $6 \mathrm{mg}$, calci carbonat: $20 \mathrm{mg}$, calci hydrophosphat (DCP): 160,7 mg, ethanol 30\% điều chỉnh đến $\mathrm{pH}$ 8,0: $300 \mathrm{mg}$. Viên không dính chày cối trong quá trình dập viên ở quy mô 1000 viên/mẻ và đạt tiêu chuẩn ĐHT theo USP 41.

Tư khóa: Acid alpha lipoic (ALA), độ hòa tan (ĐHT), dính chày cối, viên nén.

\section{SUMMARY \\ FORMULATION OF ALPHA LIPOIC ACID TABLET 300 MG}

Objectives: (i) Initially evaluating the effects of some formula factors on alpha lipoic acid (ALA) tablets' sticking phenomenon during compaction process and tablets' dissolution; (ii) Preparing 300 mg ALA tablets meeting the USP 41 requirement for dissolution test on a laboratory scale. Method: Tablets were prepared by wet-granulation method, evaluate the effects of some factors by experiments. Result: The selected fomula of ALA tablet contained: ALA: 300 mg, Avicel PH-102: 120 mg, sodium stearyl

${ }^{1}$ Trường Đại học Dược Hà Nội

Chịu trách nhiệm chính: Nguyễn Thị Thanh Duyên Email: duyenntt@hup.edu.vn Ngày nhận bài: 2.3.2021

Ngày phản biện khoa học: 22.4.2021

Ngày duyệt bài: 29.4.2021 fumarate (NSF): 19,1 mg, Aerosil 200: 19,1 mg, talcum: 9,6 mg, disodium edetate (EDTA): $1 \mathrm{mg}$, hydroxypropyl cellulose (HPC): 12,2 mg, sodium croscarmellose (NCC): $25 \mathrm{mg}$, menthol: $12 \mathrm{mg}$, poloxamer: $6 \mathrm{mg}$, calcium carbonate: $20 \mathrm{mg}$, calcium hydrogen phosphate (DCP): $160,7 \mathrm{mg}$, ethanol $30 \%$ to $\mathrm{pH} 8,0$ : 300mg. The selected formula were successfully prepared on a scale of 1000 tablets without punch sticking and met the USP 41 requirement for dissolution test.

Keywords: Acid alpha lipoic (ALA), dissolution, punch sticking, tablet.

\section{I. ĐĂT VẤN ĐỀ}

Acid alpha lipoic (ALA) là dược chất có tác dụng chống oxy hóa, thường được chỉ định để điêu trị các tổn thương gây ra bởi quá trình oxy hóa và tái tạo các chất chống oxy hóa nội sinh [2]. Tuy nhiên, việc sản xuất và bảo quản dạng thuốc viên nén chứa ALA gặp nhiêu khó khăn do dược chất có nhiệt độ nóng chảy thấp (ở $60^{\circ} \mathrm{C}$ ), ít tan trong nước, kém ổn định với nhiệt và ánh sáng, viên nén dễ bị dính chày trong quá trình dập viên, khả năng tự polyme hóa của ALA còn có thể làm cho viên nén ALA giảm hàm lượng $(\mathrm{HL})$ và $\mathrm{OHT}$ sau một thời gian sản xuất [2], [8]. Do vậy, nghiên cứu này được thực hiện với các mục tiêu: (i) Bước đâu đánh giá được ảnh hưởng của một số yếu tố thuộc công thức bào chế đến khả năng khắc phục hiện tượng dính chày cối trong quá trình dập viên và ĐHT của viên; (ii) Bào chế được viên nén ALA 300 mg đạt tiêu chuẩn ĐHT theo USP 41 ở quy mô phòng thí nghiệm.

\section{NGUYÊN LIỆU VÀ PHƯƠNG PHÁP \\ 2.1. Nguyên liệu}

Dược chất: ALA (Trung Quốc - TQ) và một số tá dược khác như: tinh bột sắn (TQ), Avicel PH102 (Đài Loan), DCP (TQ), hydroxypropyl methylcellulose (HPMC) E5 (Singapore), HPC (Singapore), natri starch glycolat (NSG) (TQ), 